\title{
Prevalencia de patología oftalmológica en prematuros menores de un año de edad
}

\author{
JUAN PABLO LÓPEZ G. ${ }^{1}$, DIEGO OSSANDÓN V. ${ }^{1}$, OLIVER DENK V. ${ }^{1}$, \\ RICARDO STEVENSON A. ${ }^{1}$, RICARDO AGURTO R. ${ }^{1}$, ANDRÉS UAUY N. ${ }^{2}$, \\ RICARDO SALINAS G. ${ }^{3}$, MARCELA PÉREZ R. ${ }^{3}$, HORACIO COX M. ${ }^{3}$, \\ ANDRÉS MATURANA P. ${ }^{3}$, SOLEDAD ELÍAS A. ${ }^{4}$ \\ 1. Oftalmólogo, Servicio de Oftalmología, Clínica Alemana de Santiago. \\ 2. Alumno de Medicina, Universidad del Desarrollo. \\ 3. Neonatólogo, Servicio de Neonatología, Clínica Alemana de Santiago. \\ 4. Enfermera Universitaria, Servicio de Medicina Preventiva, Clínica Alemana de Santiago.
}

\begin{abstract}
Prevalence of ocular pathology in premature children under one year of age

Objective: To report ocular abnormalities in premature infants examined before one year of Corrected Gestational Age (CGA). Patients and Methods: Retrospective review of medical records of a number of premature infants ([birth weight (BW) $\leq 1500 \mathrm{~g}$ and/or $\leq 32$ week gestational age (GA)] born between 2006 and 2009 and examined before one year of CGA. GA, BW, retinopathy of prematurity (ROP), CGA examination, refraction, anisometropia and strabismus information were recorded. Results: Out of the 149 premature infants born during the period, 100 had eye exam before one year of age (67.14\%), the mean GA and BW was 29.3 (range 23-36) weeks and 1217 (343-2190) g, respectively. 29\% had ROP, 4\% required treatment. The mean CGA at the time of ophthalmologic examination was 6.4 (3 to 11.5) months. The spherical equivalent was $+1.34 \mathrm{D}(-1.75$ to +5.75$), 2.2 \%$ presented anisometropia $>1 \mathrm{D}(95 \% \mathrm{CI},-0.82$ to 5.26$) .3 .3 \%(95 \% \mathrm{CI},-0.38$ to 7.04 ) were in need of glasses according to international recommendations and $4 \%$ (95\% CI, 0.16 to 7.84 ) had strabismus. Conclusions: The low prevalence of risk factors for amblyopia detected in this population compared with that reported for premature infants with severe ROP could be explained by the low prevalence of the latter in this study.
\end{abstract}

(Key words: Retinopathy of prematurity, strabismus, anisometropia).

Rev Chil Pediatr 2012; 83 (6): 570-576

\section{RESUMEN}

Objetivo: Reportar hallazgos oftalmológicos en niños prematuros examinados antes del año de edad gestacional corregida (EGC). Pacientes y Método: Revisión retrospectiva de fichas clínicas de una serie de prematuros ([peso nacimiento $(\mathrm{PN}) \leq 1500 \mathrm{~g}$ y/o $\leq 32 \mathrm{sem}$ edad gestacional (EG)] nacidos en CAS entre 2006-2009, examinados antes del año de EGC. Se consignó EG, PN, antecedente de retinopatía del prematuro (ROP), EGC

Recibido el 01 de febrero de 2012, devuelto para corregir el 12 de marzo de 2012, segunda versión el 23 de abril de 2012 , tercera versión el 06 de julio de 2012, aceptado para publicación el 28 de agosto de 2012.

Este trabajo cumple con los requisitos sobre consentimiento /asentimiento informado, comité de ética, financiamiento, estudios animales y sobre la ausencia de conflictos de intereses según corresponda.

Correspondencia a:

Juan P. López G.

E-mail: jpablolopezg@hotmail.com 
al examen, refracción, anisometropía y estrabismo. Resultados: De 149 prematuros nacidos en el período, 100 tuvieron examen oftalmológico antes del año de edad (67,14\%); el promedio de EG y PN fue 29,3 (rango 2336) semanas y 1217 (343-2190) g, respectivamente. El 29\% presentó ROP, requiriendo tratamiento el 4\%. La EGC promedio al examen oftalmológico fue 6,4 (3-11,5) meses. El equivalente esférico fue +1,34D (-1,75 a $+5,75)$, presentando anisometropía $>1 \mathrm{D}$ el 2,2\%(95\% IC, -0,82-5,26). El 3,3\%(95\% IC, -0,38-7,04) presentaba indicación de lentes según recomendaciones internacionales y el 4\%(95\% IC, 0,16-7,84) presentó estrabismo. Conclusiones: La baja prevalencia de factores de riesgo para ambliopía detectada en esta cohorte comparada con la reportada para prematuros con ROP severa podría explicarse por la baja prevalencia de ésta última en la presente serie.

(Palabras clave: Retinopatía del prematuro, estrabismo, anisometropía).

Rev Chil Pediatr 2012; 83 (6): 570-576

\section{Introducción}

Existe una conocida asociación entre prematurez y mayor frecuencia de presentación de patologías oftalmológicas dentro de las que se incluyen, además de la retinopatía del prematuro (ROP), los vicios de refracción, el estrabismo y la ambliopía ${ }^{1-6}$. Esta mayor probabilidad de presentar morbilidad oftalmológica sitúa a los niños prematuros en un grupo de especial riesgo que requiere controles clínicos seriados a fin de detectarla y tratarla a tiempo ya que de otro modo puede conducir al desarrollo anormal de la visión, fenómeno conocido como ambliopía ${ }^{7}$. No obstante, excluyendo el estricto y bien normado esquema que existe en el mundo (incluido Chile) para el screening de la ROP, el momento óptimo para realizar el primer examen oftalmológico a los niños prematuros una vez que ha cesado el riesgo de ROP es aún motivo de controversia en la literatura internacional.

En vista que la prevalencia de patología oftalmológica en los niños prematuros atendidos en nuestra institución (Clínica Alemana de Santiago (CAS), Santiago, Chile) era desconocida tanto para pediatras como oftalmólogos, quisimos investigar nuestra realidad local en cuanto a la prevalencia y tipo de patología oftalmológica de nuestros pacientes a fin de determinar el momento más apropiado para efectuar su primer examen oftalmológico de seguimiento. Con este propósito iniciamos el año 2005 un protocolo para realizar un examen oftalmológico completo a todos los niños prematuros nacidos en CAS antes de su primer año de edad gestacional corregida (EGC). El objetivo del presente trabajo es reportar los hallazgos del examen oftalmológico practicado a dicho grupo de niños prematuros y proponer recomendaciones para su primer examen.

\section{Pacientes y Método}

Se realizó una revisión retrospectiva de las fichas clínicas de una serie de niños prematuros $([$ peso nacimiento $(\mathrm{PN}) \leq 1500 \mathrm{~g}$ y/o $\leq 32$ semanas de edad gestacional (EG)] nacidos en CAS entre los años 2006 y 2009 que fueron examinados oftalmológicamente antes del año de EGC. También se incluyeron en la revisión aquellos niños de $\mathrm{PN}>1500$ g y EG $>32$ semanas que recibieron screening de ROP a solicitud expresa del neonatólogo tratante por presentar un "curso clínico inestable" según recomendaciones internacionales ${ }^{8}$. Se consignó para cada paciente la edad gestacional y el peso de nacimiento, antecedente de haber presentado retinopatía del prematuro (ROP) y algunos factores sistémicos clásicamente asociados con ROP como dependencia de oxígeno a los 28 días de vida y 36 semanas de edad postmenstrual, ductus arterioso persistente, sepsis neonatal, hemorragia intraventricular, transfusiones sanguíneas ${ }^{8}$ y algunos factores descritos como protectores de incidencia y severidad de ROP como el uso antenatal de surfactante y esteroides ${ }^{9,10}$. Esta revisión retrospectiva contó con la aprobación del Comité Científico Docente de nuestra institución (CAS). Los exámenes oftalmológicos fueron realizados por un grupo de 5 oftalmólogos especialistas en oftalmología pediátrica (2) y retina (3), participantes activos del Programa 
de Retinopatía del Prematuro del MINSAL, siendo la gran mayoría (60\%) de las evaluaciones practicadas por uno de los autores (JPLG). El examen oftalmológico se realizó de la forma estándar y habitual para niños preverbales, consistiendo en la estimación de fijación y seguimiento, examen de motilidad ocular, prisma-cover test para cerca y lejos para determinar estrabismo, exploración del segmento anterior con biomicroscopio y refracción con esquiascopia y examen de fondo de ojos con oftalmoscopia binocular indirecta, previa dilatación pupilar con colirio de tropicamida $1 \%$ y fenilefrina $2,5 \%$, siguiendo el Anexo 2 del protocolo de la Guía Clínica de Retinopatía del Prematuro, MINSAL $2010^{11}$.

Para el examen oftalmológico se consignó la edad gestacional corregida al momento del examen, la refracción ciclopléjica, presencia de anisometropía (diferencia entre el estado refractivo de ambos ojos) y estrabismo. La refracción promedio de los niños con astigmatismo se calculó como equivalente esférico cuyo valor en dioptrías (D) corresponde a la suma del vicio de refracción esférico (miopía o hipermetropía) más la mitad del valor del cilindro astigmático. Los vicios de refracción se categorizaron siguiendo la clasificación utilizada por Holmström ${ }^{5}$, considerando cualquier miopía menor a $0 \mathrm{D}$, hipermetropía mayor a 3 $\mathrm{D}$ y astigmatismo mayor a $1 \mathrm{D}$. No obstante, los vicios de refracción y el grado de anisometropía fueron considerados como significativos (capaces de generar ambliopía), aplicando las recomendaciones de consenso de expertos de la Academia Americana de Oftalmología Pediátrica y Estrabismo (AAPOS) para la evaluación de niños preverbales ${ }^{12}$, según se detalla: miopía mayor a $-5 \mathrm{D}$, hipermetropía mayor a $+6 \mathrm{D}$, astigmatismo mayor a $3 \mathrm{D}$ (o mayor a $1 \mathrm{D}$ si el eje es oblicuo) y anisometropía mayor a $1 \mathrm{D}$ de equivalente esférico. Todo estrabismo manifiesto fue considerado significativo. En algunos casos se estimó la agudeza visual mediante un test de mirada preferencial (test de Teller), que no constituía un examen de rutina en el protocolo inicial de examen de seguimiento oftalmológico de niños prematuros, siendo solicitado, por consiguiente, sólo a discreción del oftalmólogo examinador.
Se calcularon intervalos de confianza para cada variable y las diferencias de prevalencias de patología asociada (sistémica y oftalmológica) entre niños con ROP $v s$ sin ROP fueron estimadas con la prueba de $\chi^{2}$ (Stats Calculator, KT crearinghouse, http://ktclearinghouse. $\mathrm{ca} /$ cebm/practise/ca/calculators/statscalc). La comparación entre variables contínuas (PN y EG) se realizó utilizando la prueba de T-de Student disponible en http://studentsttest.com/. La potencia de nuestra muestra para la detección de miopía y estrabismo en niños prematuros se estimó en un $88 \%$ y $87 \%$, respectivamente, utilizando como referencia poblacional los estudios de Bremer ${ }^{13}$ y Quinn ${ }^{19}$. Se consideró significancia estadística a un $\mathrm{p}<0,05$.

\section{Resultados}

De un total de 149 niños prematuros nacidos en el período del estudio se identificaron $100(67,14 \%)$ a quienes se les había realizado un examen oftalmológico de seguimiento antes del año de EGC, siendo el $50 \%$ de sexo femenino. El promedio de EG y PN fue de 29,3 semanas (rango 23-36) y 1217 g (rango 343-2 190), respectivamente, incluyéndose en la revisión 3 niños sin criterio clásico para screening ROP $(\mathrm{PN}>1500$ g y EG > 32 semanas) que recibieron screening a solicitud de su neonatólogo tratante por presentar un "curso clínico inestable".

El 29\% de los niños presentó ROP, correspondiendo el $48,3 \%$ de los casos a la etapa 1 de la enfermedad, el $24,1 \%$ a etapa 2 y el $27,6 \%$ a etapa 3. Sólo 4 niños (4\%) requirieron tratamiento con LASER y ninguno necesitó cirugía para reparación de desprendimiento de retina (vitrectomía). El PN y la EGC promedio para los niños con ROP fue de 860,2 $\pm 283,16 \mathrm{~g}$ y $26,86 \pm 2,16$ semanas respectivamente, difiriendo significativamente de los niños sin ROP que presentaron un PN de $1362,52 \pm 350,84 \mathrm{~g}$ $(\mathrm{p}<0,001)$ y EGC $30,37 \pm 1,88$ semanas $(\mathrm{p}<0,001)$. La patología sistémica más frecuentemente asociada según el antecedente de ROP se muestra en la tabla 1, alcanzando significancia estadística el uso de oxígeno a los 28 días de vida y 36 semanas de edad postmens- 
Tabla 1. Asociación entre patología sistémica y antecedente de retinopatía del prematuro en 97 niños prematuros

\begin{tabular}{|c|c|c|c|}
\hline \multirow[b]{2}{*}{$\begin{array}{l}\text { Patología sistémica } \\
\text { asociada }\end{array}$} & \multicolumn{3}{|c|}{$N^{\circ}$ de niños con antecedente de ROP } \\
\hline & $\begin{array}{c}\text { Con } \\
(n=29)\end{array}$ & $\begin{array}{c}\text { Sin } \\
(n=68)\end{array}$ & p-Value \\
\hline $\begin{array}{l}\text { Requerimiento de oxígeno } \\
\text { A las } 36 \text { semanas } \\
\text { A los } 28 \text { días }\end{array}$ & $\begin{array}{l}15 \\
22\end{array}$ & $\begin{array}{l}10 \\
15\end{array}$ & $\begin{array}{l}<0,001 \\
<0,001\end{array}$ \\
\hline Ductus arterioso persistente* & 19 & 23 & 0,005 \\
\hline Sepsis neonatal & 7 & 9 & 0,305 \\
\hline Hemorragia intraventricular* & 1 & 3 & 0,702 \\
\hline Transfusiones sanguíneas & 20 & 19 & $<0,001$ \\
\hline Uso surfactante & 22 & 25 & 0,001 \\
\hline Uso prenatal esteroides & 25 & 54 & 0,615 \\
\hline
\end{tabular}

*Para estas características se contaba con el dato en 100 niños de la serie.

Tabla 2. Prevalencia de estrabismo, vicios de refracción y anisometropía significativos según antecedente de retinopatía del prematuro

\begin{tabular}{|lccc|}
\hline $\begin{array}{l}\text { Antecedente } \\
\text { de ROP }\end{array}$ & $\begin{array}{c}\text { Estrabismo } \\
\mathbf{N}(\mathbf{n}=\mathbf{1 0 0})\end{array}$ & $\begin{array}{c}\text { Vicios de } \\
\text { refracción } \\
(\mathbf{n}=\mathbf{9 0})\end{array}$ & $\begin{array}{c}\text { Anisometropía } \\
(\mathbf{n}=\mathbf{9 0})\end{array}$ \\
$\operatorname{con}(n=29)$ & 2 & 1 & 0 \\
$\sin (n=71)$ & 2 & 2 & $2^{*}$ \\
$p$-Value & 0,702 & 0,608 & 0,912 \\
\hline
\end{tabular}

*Un paciente presentaba anisometropía y astigmatismo significativo en forma simultánea. ROP: retinopatía del prematuro.

trual, el antecedente de ductus arterioso persistente, transfusiones sanguíneas y uso de surfactante antenatal.

La EGC promedio al momento del examen oftalmológico fue de 6,4 (rango 3-11,5) meses. El 60\% de los niños tuvo estimación de visión con test de Teller, encontrándose fuera de límites normales para la edad en 4 niños $(6,7 \%)$ de los cuales sólo uno presentaba patología oftalmológica significativa (estrabismo). La información referente a refracción ciclopléjica se encontraba consignada para 90 niños de la serie en quienes la refracción promedio (equivalente esférico) para ambos ojos fue de $+1,34 \mathrm{D}$ (rango - 1,75 a $+5,75$ ). Se observó miopía (cualquier nivel) en el 6,6\% de los pacientes, hipermetropía mayor a $3 \mathrm{D}$ en el $11,1 \%$ y astigmatismo mayor a $1 \mathrm{D}$ en el $32 \%$. El 2,2\%(95\% IC, $-0,82-5,26)$ de los niños presentó una anisometropía mayor a 1 D. No obstante, los vicios de refracción considerados significativos como para proceder a la indicación de uso de lentes sólo se presentaron en nuestra serie en el 3,3\% (95\% IC, -0,38-7,04) de los niños con exámenes de refracción disponible, correspondiendo a 3 casos de astigmatismo oblicuo.

El examen de estrabismo se encontraba consignado para los 100 niños de la serie, encontrándose alterado en el $4 \%$ de ellos $(95 \%$ IC, 0,16-7,84), correspondiendo a 3 casos de exotropia y un caso de endotropia.

La prevalencia de vicios de refracción, anisometropía y estrabismo significativos no difirió significativamente entre los niños con antecedentes de ROP y sin ROP (tabla 2). De los 4 niños que recibieron tratamiento con LASER por presentar ROP de alto riesgo en ambos ojos ninguno presentó estrabismo y en los 3 casos en que se contaba con la refracción consignada no se encontró anisometropía o vicio de refracción significativo. El cuarto niño continuó sus controles en otro centro, examinándose por nuestro grupo sólo recientemente (Enero 2012), no presentando patología oftalmológica, pero se desconoce su estado refractivo previo. Al analizar los 8 niños que presentaron alguna patología oftalmológica significativa (tabla 2) encontramos que sólo 3 de ellos tenían antecedente de haber presentado ROP; en los 3 casos correspondió a ROP más avanzada (etapa 3) y ninguno requirió tratamiento con LASER. Los 5 restantes no tenían antecedente de ROP y no se encontró mayor prevalencia de alguna patología específica asociada en este grupo.

\section{Discusión}

La ambliopía u "ojo flojo", principal causa de mala visión en los niños, consiste en la disminución de la visión que ocurre cuando, por diversos factores como los vicios de refracción, la anisome- 
Tabla 3. Prevalencia de estrabismo, vicios de refracción y anisometropía en algunas series de niños prematuros publicadas en la literatura

\begin{tabular}{|c|c|c|c|c|c|c|c|c|}
\hline \multicolumn{2}{|c|}{ Autor/estudio (año) } & \multirow{2}{*}{$\begin{array}{c}\begin{array}{c}\text { n } \\
\text { niños }\end{array} \\
468\end{array}$} & \multirow{2}{*}{$\begin{array}{c}\text { \% } \\
\text { ROP }\end{array}$} & \multirow{2}{*}{$\begin{array}{c}\text { Edad al } \\
\text { examen } \\
\text { (meses) } \\
6\end{array}$} & \multirow{2}{*}{$\begin{array}{c}\% \\
\text { estrabismo } \\
6,4\end{array}$} & \multirow{2}{*}{$\begin{array}{c}\text { \% vicios de } \\
\text { refracción } \\
\text { N/E }\end{array}$} & \multirow{2}{*}{\multicolumn{2}{|c|}{$\begin{array}{c}\% \\
\text { anisometropía } \\
\mathrm{N} / \mathrm{E}\end{array}$}} \\
\hline Laws $^{18}$ & (1992) & & & & & & & \\
\hline Cryo ROP13 & (1998) & 2449 & 69 & 12 & 11,8 & N/E & & $\mathrm{N} / \mathrm{E}$ \\
\hline $\begin{array}{l}\text { ET-ROP14 } \\
\text { Alto riesgo } \\
\text { Bajo Riesgo }\end{array}$ & (2006) & $\begin{array}{l}366 \\
306\end{array}$ & $\begin{array}{l}100 \\
100\end{array}$ & $\begin{array}{l}6 \\
6\end{array}$ & $\begin{array}{r}20,3 \\
9,6\end{array}$ & $N / E$ & & $\mathrm{~N} / \mathrm{E}$ \\
\hline Quinn/ Cryo ROP ${ }^{19}$ & $(1992)$ & 2626 & 63 & 12 & N/E & $\begin{array}{c}22 \\
\text { (Miopía }<-0,25 D)\end{array}$ & 3,35 & $5(>2 D)$ \\
\hline Holmstrom 5 & (1998) & 247 & 38 & 6 & $N / E$ & $\begin{array}{r}8 \text { (Miopía < 0D) } \\
12,5(\mathrm{HM}>+3 \mathrm{D}) \\
51,8(\text { Astig > 1D) }\end{array}$ & 6,6 & $(>1 \mathrm{D})$ \\
\hline $\begin{array}{l}\text { Davitt/ET ROP20 } \\
\text { Alto riesgo tratados } \\
\text { Alto riesgo, manejo }\end{array}$ & $\begin{array}{l}\text { (2005) } \\
\text { o convencional }\end{array}$ & $\begin{array}{l}321 \\
307\end{array}$ & $\begin{array}{l}100 \\
100\end{array}$ & 9 & $N / E$ & $\begin{array}{c}\text { (Mopía < -0,25D) } \\
64,5 \\
69,4\end{array}$ & & $\mathrm{~N} / \mathrm{E}$ \\
\hline Larsson ${ }^{4}$ & (2006) & 198 & 40 & 6 & N/E & 55 (Astig >1D) & 7,6 & $(>1 \mathrm{D})$ \\
\hline López JP. et al & & 100 & 29 & 6,4 & 4 & $\begin{array}{c}6,1(\text { Miopía < OD) } \\
11,1(\mathrm{HM}>+3 \mathrm{D}) \\
32(\text { Astig }>1 \mathrm{D})\end{array}$ & 2,2 & $(>1 \mathrm{D})$ \\
\hline
\end{tabular}

$\mathrm{N} / \mathrm{E}=$ no estudiado; $\mathrm{HM}=$ hipermetropía; Astig = astigmatismo; $\mathrm{ROP}=$ retinopatía del prematuro; $\mathrm{D}=$ dioptrías.

tropía, el estrabismo y la deprivación visual, uno o ambos ojos envían una imagen borrosa o mal definida al cerebro. Si la ambliopía no es tratada en forma oportuna en la infancia la pérdida de visión puede ser permanente para el resto de la vida adulta ${ }^{7}$. Existe abundante evidencia bibliográfica sobre el mayor riesgo de los niños prematuros de presentar patología oftalmológica significativa que puede conducir a la aparición de ambliopía, siendo mayor el riesgo a menor peso y/o edad gestacional de nacimiento y mayor también en presencia $\mathrm{y}$ severidad de $\mathrm{ROP}^{1,3,4}$. Este riesgo ha sido más estudiado para el estrabismo que para los vicios de refracción y anisometropía significativos ${ }^{13-16}$. Es por esta razón que los niños prematuros deben recibir un seguimiento oftalmológico durante toda la infancia a fin de detectar los factores de riesgo de ambliopía descritos. No obstante, las recomendaciones internacionales sobre el inicio, frecuencia y suspensión de los controles de seguimiento oftalmológico en niños prematuros son muy variables ${ }^{1,15}$.

Existen escasos reportes en la literatura sobre prevalencia de patología oftalmológica en niños prematuros menores de un año (ta- bla 3). En Chile se cuenta con una Guía Clínica sobre Retinopatía del Prematuro, MINSAL 2010, para el diagnóstico y tratamiento de la $\mathrm{ROP}^{11}$ donde se señala en su página 17 que los prematuros que no presentaron ROP o que la tuvieron en bajo riesgo (Tipo 2) requieren un control anual hasta los 4 años, pero no señala en qué momento debe realizarse el primer examen oftalmológico una vez que los niños han sido dados de alta del screening para ROP. El mismo grupo del MINSAL ha publicado un análisis epidemiológico de los recién nacidos con menos de 32 semanas en la red pública de salud de Chile, Quinquenio 2000-2004 ${ }^{17}$, donde se señala contar con datos sobre prevalencia de estrabismo $(6,5 \%)$ y miopía $(1,23 \%)$ a los 2 años de edad en prematuros egresados de 5 centros del país pero no se entrega información sobre los criterios utilizados para su clasificación ni sobre los hallazgos del examen oftalmológico antes de dicha edad.

En nuestra serie se encontró una baja proporción de niños prematuros menores a un año de edad con patología oftalmológica significativa capaz de producir ambliopía $(2,2 \%$ de anisometropía, 3,3\% de vicios de refracción y 4\% 
de estrabismo), siendo estos valores menores a los reportados en la literatura internacional para niños prematuros de similares características (tabla 3). Tampoco encontramos que la patología oftalmológica se concentrara en los 4 niños que presentaron la ROP más grave y que requirieron tratamiento. No obstante, la mayor prevalencia de miopía y estrabismo en prematuros con ROP de mayor gravedad es un hecho ampliamente conocido y reportado en la literatura, especialmente si estos niños han recibido tratamiento con LASER o crioterapia $^{3,5,16,18-21}$, por lo que el bajo número de pacientes en nuestra serie con ROP grave podría explicar las bajas cifras de patología oftalmológica encontrada. Del mismo modo, un seguimiento a más largo plazo podría hacer evidente la aparición de patología oftalmológica en estos niños en los controles sucesivos.

Dentro de las fortalezas de nuestro estudio destacan el hecho de ser el primer estudio nacional donde se reportan hallazgos oftalmológicos para prematuros menores de un año. Por otra parte, consideramos haber obtenido un tamaño de muestra que refleja una buena aproximación a lo que ocurre en nuestra "realidad" clínica en las mejores condiciones posibles para una revisión retrospectiva ya que este estudio se realizó en una institución de salud privada con buena cobertura tanto para los exámenes pediátricos como oftalmológicos.

Nuestro estudio presenta también importantes debilidades inherentes a los estudios retrospectivos como son la pérdida importante de seguimiento de los pacientes que alcanzó un $33 \%$, la presencia de diferentes oftalmólogos que realizaron los exámenes y las diferentes edades de los pacientes al momento del examen. Asimismo, desconocemos como variará en el tiempo la prevalencia de la patología oftalmológica en esta cohorte por lo que un seguimiento a largo plazo será necesario para obtener esta información. Por último, tampoco consignamos en nuestros pacientes factores raciales ni antecedentes familiares de patología oftalmológica lo que podría constituir un elemento diferenciador de nuestra muestra con respecto a otras series de niños prematuros atendidos en Chile en el sistema público de salud. Un estudio presentado durante el II
Latin American Workshop on Retinopathy Of Prematurity $^{22}$, en que se comparó una serie de 124 niños prematuros nacidos en un hospital público del área oriente de Santiago con 167 nacidos en CAS no mostró diferencias estadísticamente significativas en cuanto a PN, EG, incidencia y tipo de ROP y necesidad de tratamiento entre ambos grupos por lo que postulamos que probablemente la prevalencia de patología oftalmológica no debería diferir significativamente entre ambos grupos de niños. Un estudio similar al presente realizado en niños prematuros de otras instituciones podría permitir confirmar esta sospecha clínica.

Considerando que cada examen oftalmológico representa una visita adicional y un agregado factor de estrés para este grupo de niños y sus familias, y dada la ausencia hasta la fecha de un consenso internacional respecto a la edad ideal para comenzar con los exámenes oftalmológicos de los niños prematuros, nuestros datos indican que parecería ser prudente a fin de lograr la máxima detección de factores de riesgo de ambliopía con un mínimo de exámenes el iniciar los exámenes oftalmológicos de seguimiento de los niños prematuros de bajo riesgo oftalmológico, es decir, que no hayan presentado una ROP severa (etapa 2 ó 3), a la edad de un año de EGC. No obstante, el diseño metodológico del presente estudio no permite concluir esta observación ni mucho menos generalizar nuestros resultados a niños prematuros atendidos en otras instituciones.

En suma, la baja prevalencia de ROP de alto riesgo en nuestros pacientes podría explicar a su vez la baja prevalencia de factores de riesgo de ambliopía detectados en esta cohorte.

\section{Agradecimientos}

Los autores agradecen especialmente al Dr. Sergio Galano Triviño, Oftalmólogo de Clínica Alemana de Santiago, por su invaluable ayuda en el estudio estadístico y análisis de los resultados.

\section{Referencias}

1.- O'Connor AR, Stewart CE, Singh J, Fielder AR: Do infants of birth weight less than $1500 \mathrm{~g}$ require additional 
long term ophthalmic follow up? Br J Ophthalmol 2006; 90 (4): 451-5.

2.- Cats BP, Tan KE: Prematures with and without regressed retinopathy of prematurity: comparison of long-term (6-10 years) ophthalmological morbidity. J Pediatr Ophthalmol Strabismus 1989; 26 (6): 271-5.

3.- Saunders KJ, McCulloch DL, Shepherd AJ, Wilkinson $A G$ : Emmetropisation following preterm birth. $\mathrm{Br} \mathrm{J}$ Ophthalmol 2002; 86 (9): 1035-40.

4.- Larsson EK, Holmström GE: Development of astigmatism and anisometropia in preterm children during the first 10 years of life: a population-based study. Arch Ophthalmol 2006; 124 (11): 1608-14.

5.- Holmström M, el Azazi M, Kugelberg U: Ophthalmological long-term follow up of preterm infants: a population based, prospective study of the refraction and its development. Br J Ophthalmol 1998; 82 (11): 1265-71.

6.- Morrison DG, Emanuel M, Donahue SP: Risk of refractive pathology after spontaneously regressed ROP in emmetropic patients. J Pediatr Ophthalmol Strabismus 2010; 47 (3): 141-4.

7.- Holmes JM, Clarke MP: Amblyopia. Lancet 2006; 367 (9519): 1343-51.

8.- Anón: Screening examination of premature infants for retinopathy of prematurity. Pediatrics 2006; 117 (2): 572-6.

9.- Higgins RD, Mendelsohn AL, DeFeo MJ, Ucsel R, Hendricks-Munoz KD: Antenatal dexamethasone and decreased severity of retinopathy of prematurity. Arch Ophthalmol 1998;116 (5): 601-5.

10.- Holmes JM, Cronin CM, Squires P, Myers TF: Randomized clinical trial of surfactant prophylaxis in retinopathy of prematurity. J Pediatr Ophthalmol Strabismus 1994; 31 (3): 189-91.

11.- Guía Clínica Retinopatía del Prematuro, Serie Guías Clínicas MINSAL, 2010. Available at: http://www. minsal.gob.cl/portal/url/item/721fc45c973b9016e0400 1011f0113bf.pdf.

12.- Miller JM, Harvey EM: Spectacle prescribing recommendations of AAPOS members. J Pediatr Ophthalmol Strabismus 1998; 35 (1): 51-2.

13.- Bremer DL, Palmer EA, Fellows RR, et al: Strabismus in premature infants in the first year of life. Cryotherapy for Retinopathy of Prematurity Cooperative Group. Arch. Ophthalmol 1998; 116 (3): 329-33.

14.- VanderVeen DK, Coats DK, Dobson V, et al: Prevalence and course of strabismus in the first year of life for infants with prethreshold retinopathy of prematurity: findings from the Early Treatment for Retinopathy of Prematurity study. Arch Ophthalmol 2006; 124 (6): 766-73.

15.- Holmström G, Larsson E: Long-term follow-up of visual functions in prematurely born children--a prospective population-based study up to 10 years of age. $\mathrm{J}$ AAPOS 2008; 12 (2): 157-62.

16.- Yang C-S, Wang A-G, Sung $C-S$, et al: Long-term visual outcomes of laser-treated threshold retinopathy of prematurity: a study of refractive status at 7 years. Eye (Lond) 2010; 24 (1): 14-20.

17.- Informe Técnico Recién Nacidos con menos de 32 semanas de edad gestacional. Sistema Nacional de Servicios de Salud de Chile. Quinquenio 2000-2004. Available at: http://www.prematuros.cl/webmarzo08/ InformeRN.pdf.

18.- Laws D, Shaw DE, Robinson J, et al: Retinopathy of prematurity: a prospective study. Review at six months. Eye (Lond) 1992; 6 (Pt 5): 477-83.

19.- Quinn GE, Dobson V, Repka MX, et al: Development of myopia in infants with birth weights less than 1251 grams. The Cryotherapy for Retinopathy of Prematurity Cooperative Group. Ophthalmology 1992; 99 (3): 32940.

20.- Davitt BV, Dobson V, Good WV, et al: Prevalence of myopia at 9 months in infants with high-risk prethreshold retinopathy of prematurity. Ophthalmology. 2005; 112 (9): 1564-8.

21.- Quinn GE, Dobson V, Davitt BV, et al: Progression of myopia and high myopia in the early treatment for retinopathy of prematurity study: findings to 3 years of age. Ophthalmology 2008; 115 (6): 1058-64.e1.

22.- López JP et al: Retinopatía del Prematuro en un Hospital Público de Santiago de Chile. Presentado como poster en The Second Latin American Workshop on Retinopathy of Prematurity, Mar del Plata, Argentina, 2008. Available at: http://www.v2020la.org/bulletin/esp/ docs/boletin_16/docs/SIBEN.pdf. 